\title{
The relationship between stress and negative emotion: The Mediating role of rumination
}

\author{
Jiaxuan Du, Jiali Huang, Yuanyuan An and Wei Xu* \\ School of Psychology, Nanjing Normal University, Nanjing, P.R. China
}

\begin{abstract}
Using ambulatory assessment, the current study investigated the dynamic impact of stress on negative emotions in daily life and explored the mediating role of rumination in this relationship. A total of 100 college students were recruited and reported their perceived stress, current ruminative thinking and negative emotions (including anxiety, depression, and anger) 5 times a day for 7 consecutive days. Mixed model analyses indicated that the higher level of stress at the current time predicted increased negative emotions at the next time including depression, anger and anxiety. At within-person level, rumination significantly mediated the relationship of stress to depression, anger, and anxiety. At between-person level, overall rumination significantly mediated the relationship between stress and angry. Findings suggested that people who perceived higher level stress may report more rumination, which in turn may lead to increases in negative emotions.
\end{abstract}

\section{Introduction}

Individuals may encounter a variety of stress events in daily life. If those events are disposed inappropriately, there will be a high probability of producing a series of negative consequences. Findings from previous studies have shown that psychological stress responses often include negative emotions. In other words, people experience a complex array of negative emotions, such as depression, anxiety, anger, and distress during real-life stressful events [1-3]. Those negative emotions may potentially lead to poorer health outcomes and subsequent increased risk of complications [4], a decreased quality of life [5], and increased health care usage [6].

Many studies have discussed the relationship between stress and negative emotions. However, understanding the mechanisms of stress associated with negative emotions is critical, as it has the potential to provide an opportunity for those in stress to reduce their negative emotions. The current study aims to investigate the association between stress and negative emotions in a daily-life context, and further to explore the potential mechanism by considering the role of rumination.

\section{Stress and Negative Emotions}

Stress is defined as a relationship between individuals and environment that is appraised as personally significant and as taxing or exceeding resources for coping [7]. According to Stress and coping theory [8] a framework is provided to testing hypotheses about the stress process and its relation to physical and mental health. Certain emotions like anger, shame, and anxiety usually arise from stress, which refers to harmful, threatening, or challenging conditions, showing a relationship between stress and emotion. Based on Lazarus and Folkman's theory [8], stress and emotion depend on how an individual evaluates (appraises) transactions with the environments. During the appraisal, when people find something significant to self is uncontrollable, they tend to feel high levels of stress.

In terms of the relationship between stress and negative emotions, researchers have reached a consensus that they are closely associated with each other. For instance, some researchers found that depression is a form of stress response [9-10]. Fiedler, et al. [11] found that stress can positively predict anxiety symptoms. What's more, a study on 5236 college students found that the higher level of stress on learning, interpersonal relationship and other issues they are facing, the more likely they are to show angry emotions. Another study on 939 American teenagers also reported that individuals' perceived stress positively predicts their anger, which in turn affects their misbehavior, such as substance use [12]. Also, the perceived stress in cancer patients indicated a significant positive correlation to anger and depression [13].

However, most of previous studies have used questionnaires as measure method. Retrospective and heuristic biases of traditional assessment instruments are known to systematically distort recollections of past experiences and events. To minimize them, Ambulatory Assessment (AA) was recently raised. AA is a method used to repeated sampling of thoughts, feelings, or behaviors as close in time to the experience as possible in the naturalistic environment [14]. It can provide real-time (or near real-time) assessments and create more stable estimates of phenomena that fluctuate over time compared to single time-point measurement [15].

As mentioned above, appraisals in stress process generate emotions that vary in quality and intensity, and numerous studies proved the association between stress and negative emotions. However, few of them examined the cognitive style as the mechanism in this relationship. Rumination, as one of the most typical negative cognitive styles, will be explored to investigate its role in the relationship between stress and negative emotions.

Correspondence to: Wei Xu, School of Psychology, Nanjing Normal University, Nanjing, P.R. China, Tel: +86-25-83598362; E-mail: livingxw@163.com

Key words: stress, negative emotions, ambulatory assessment, moderating effect

Received: January 12, 2018; Accepted: January 29, 2018; Published: January 31, 2018 


\section{Rumination and Negative Emotions}

Rumination has evolved as a construct of growing interest among researchers over the past decades. Fritz [16] defined rumination as the thinking about distressing experiences, the searching for the meaning of negative events and the thinking about what can be done to change the situation in relation to negative events. Rumination has been described as having both state and trait components $[17,18]$. The current study pays attention to its state component, which means focusing on current feelings and negative thoughts.

As part of Response Styles Theory, rumination is considered as a cognitive style associated with negative, chronic, and persistent thoughts about threats to the self. Within this theory, ruminative thinking is a pattern that individual's attention, thoughts, and behaviors are focused on the negative emotional state [19]. Researchers found when rumination was partly inhibited, the perceived distress may also be decreased [20]. Therefore, rumination is a vital factor that cause negative emotions.

According to Broderick [21], rumination aggravates depression and other negative affective states. Still other studies examining rumination in individuals supported this conclusion and demonstrated rumination to be a risk factor for depression [22-24]. Previous studies also found that rumination increased anger [25,26]and predicted anxiety symptoms [27]. In addition, Muris, et al. [28] found the results indicated that rumination was positively linked to both anxiety and depression. Therefore, rumination can be considered as a probable mechanism for the development of negative emotions.

\section{Stress and Rumination}

It is demonstrated by Nolen-Hoeksema, et al. [19] that rumination in response to stress is associated concurrently with depressive symptoms. Many researchers believed that information processing impairments contribute to ruminative tendencies [29-31], when individuals encounter of stress, cognitive control impairments may contribute to higher levels of rumination. Another study has similar conclusion that rumination is a common response to stress in adolescence [32]. In an experimental study, Smith and Alloy found that individuals' rumination was reactive to their perceived stress [33].

\section{The Current Study}

Since rumination is a main indicator of negative emotions and can also be predicted by stress, it is probable that rumination acts as a mediator in the relationship between stress and negative emotion. This mediation model will be tested in the current study. Individuals with higher perceived stress may have more rumination and more negative emotions in daily life, and more rumination would predict increased negative emotional responses.

As mentioned above, to eliminate the retrospective and heuristic biases of self-report questionnaires and investigate the dynamic relations between different variables, we adopted AA to gather individuals' data of stress, rumination, and negative emotions. In line with previous research and theory, we predicted that stress and negative emotions would be associated over time. We hypothesized that (1) stress at time $t$ predicts subsequent state negative emotions at time $t+1$ in daily life, (2) state rumination is positively associated with state negative emotions at time $t$, and (3) rumination is a mediator of the association between the relationship of stress and negative emotions at both within-person and between-person levels.

\section{Materials and Methods}

\section{Participants}

All participants were recruited from a Chinese university in this study. Recruitment was conducted through advertising in the university. A total of 100 students completed the study with an average age of 20.64 years $(S D=2.79)$. Forty-five of them $(45 \%)$ were male. Of these, 86 percent $(\mathrm{N}=86)$ were undergraduate students, 14 percent $(\mathrm{N}=14)$ were postgraduate students. None of the participants reported any history of mental disorders.

\section{Procedures}

The participants were requested to attend a laboratory session after agreeing to participate in the study, in order to know the study process detailed. The survey was programmed based on a stratified interval scheme. Participants were required to complete a questionnaire, which measured the stress, rumination, and negative emotions (i.e., depression, anger, and anxiety), five times a day (at 9 am, $11 \mathrm{am}, 3: 00$ $\mathrm{pm}$, 5:00 pm and 8:00 pm) lasting one week. The questionnaire that accessed via a web link was sent to the participants' WeChat (a popular Chinese social media and messaging platform) by the researchers at scheduled times every day. The participants were required to complete the questionnaire within one hour after they received the link on the WeChat. The participants who completed the one-week assessment would get $10 \mathrm{RMB}$.

\section{Measures}

\section{Stress}

Stress was assessed by two questions: "Do you feel nervous and stress at the moment?", and "Do you have the feeling that you can't control the important things in your life?". Item was rated on a 7-point Likert scale ranging from 0 ("not at all") to 6 ("very much so"). Higher score indicates higher level of stress. Items assessed one's feeling of stress at that moment. The questionnaire had good internal consistency reliability in the current ample, with a Cronbach's alpha of 0.81 .

\section{Rumination}

Rumination was assessed by an item: "I'm recalling all my shortcomings, failures and the things that I did wrong". Item was rated on a 7-point Likert scale ranging from 0 ("not at all") to 6 ("very much so"). Higher score indicates stronger ruminative tendencies. This item indicated the attention focused on one's momentary problems.

\section{Negative Emotions}

Negative emotions were assessed with three items assessing depression, anger, and anxiety respectively (e.g. "Do you feel angry at the moment?"). Items were rated on a 7-point Likert scale ranging from 0 ("not at all") to 6 ("very much so"). Higher scores indicate high level of negative affect. The questionnaire had good internal consistency reliability in the current ample, with a Cronbach's alpha of 0.87 .

\section{Data Analysis}

To investigate the relationships among stress, rumination and negative emotions, the HLM 7.0 was used to analyze the data. Given that a traditional Hierarchical Linear Models (HLM)-based approach to test multilevel mediation is prone to confound within-group mediation effects with between-group mediation effects, we followed Zhang, et al.'s [34] multilevel mediation approach of "centered within context with reintroduction of the subtracted means," also called CWC 
(M), to test the mediation hypotheses. All the variables in this study were measured at level 1 (within-person), therefore we analyzed the association between stress, rumination, and negative emotion (i.e., depression, anger, and anxiety) by testing 1-1-1 multilevel model. According to 1-1-1 CWC (M) approach, we test the multilevel model in 3steps. The first step tested the association between stress and negative emotion (e.g., anger score) by group-mean centering the stress (centered at each person's mean to ensure that it featured within-person fluctuations instead of between-person differences) and including the group mean of stress as a control variable in the Level 2 equation.

\section{MODEL 1}

\section{Level 1:}

$\mathrm{NE}_{-(t+1) i}=\pi_{0 i}+\pi_{1 i}^{*}\left(\right.$ STRESS $\left._{\mathrm{ti}}\right)+e_{t i}$

Level 2:

$\pi_{0 i}=b_{00}+b_{01}{ }^{*}$ STRESS $\left._{. \mathrm{i}}\right)+u_{0 i}$

$\pi_{1 i}=b_{10}$

The testing of predictions of stress for negative emotions (i.e., depression, anger, and anxiety) was divided into two levels. The level 1 was the prediction of STRESS for $_{\mathrm{ti}} \mathrm{NE}_{-(\mathrm{t}+1) \mathrm{i}}$. STRESS $\mathrm{ti}_{\mathrm{i}}$ refers to the momentary stress of a person $\mathrm{i}$ at time $t$. $\mathrm{NE}_{-(\mathrm{t}+1) \mathrm{i}}^{-(t+1)}$ refers to the momentary negative emotion of a person $i$ at time $t+1$, which was created on the basis of $\mathrm{NE}_{\mathrm{ti}}$ indicating the next time $(t+1)$ momentary negative emotion of person i. As noted in the model, $\mathrm{NE}_{-(\mathrm{t}+1) \mathrm{i}}$ was predicted

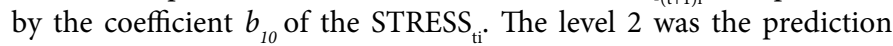
of STRESS (the average level of each individual's stress) for negative emotions. The coefficient $b_{01}$ indicated the level of individual's overall stress predicting negative emotions. The error terms $e_{t i}$ and $u_{0 i}$ captured the residual variances at Level 1 and Level 2, respectively.

The second step examined the association between stress and rumination by group-mean centering the stress and including the group mean of stress in the equation at level 2.

\section{MODEL 2}

\section{Level 1:}

RUMINATION $_{(t+1) i}=\pi_{0 i}+\pi_{1 i}^{*}\left(\right.$ STRESS $\left._{\mathrm{ti}}\right)+e_{t i}$

\section{Level 2:}

$$
\begin{aligned}
& \left.\pi_{0 i}=b_{00}+b_{01}{ }^{*}{ } \text { STRESS }_{\mathrm{i}_{\mathrm{i}}}\right)+u_{0 i} \\
& \pi_{1 i}=b_{10}
\end{aligned}
$$

The examining of predictions of stress for rumination was also divided into two levels: the predicting of stress at time $t$ for rumination at time $t+1$ and the predicting of overall stress for rumination.

The third step tested the mediating effect of rumination between stress and negative emotions by group-mean centering the stress and rumination, as well as adding the group means of the stress and rumination in the equation at level 2.

\section{MODEL 3}

Level 1:

$\mathrm{NE}_{(t+1) i}=\pi_{0 i}+\pi_{1 i}^{*}\left(\right.$ STRESS $\left._{\mathrm{ti}}\right)+\pi_{2 i}^{*}\left(\right.$ RUMINATION $\left._{(t+1) i}\right)+e_{t i}$

Level 2:

$$
\left.\pi_{0 i}=b_{00}+b_{01}{ }^{*}\left(\text { RUMINATION }_{\mathrm{i}_{\mathrm{i}}}\right)+b_{02}{ }^{*}{ } \text { STRESS }_{-\mathrm{i}}\right)+u_{0 i}
$$

$$
\begin{aligned}
& \pi_{1 i}=b_{10} \\
& \pi_{2} i=b_{20}
\end{aligned}
$$

In the model 3 , the coefficient $b_{01}$ indicated the prediction of overall rumination for negative emotions and the coefficient $b_{02}$ indicated the prediction of overall stress for negative emotions by controlling the prediction of overall rumination for negative emotions. The coefficient $b_{10}$ represented the predication of stress at time $t$ for negative emotions at time $t+1$ and $b_{20}$ represented the prediction of rumination at time $t+1$ for negative emotions at time $t+1$, which investigates the dynamic relationships between stress, rumination and negative emotions.

\section{Results}

There were 100 students participating in the research. Each participantas required to send the feedback five times a day lasting one week. Therefore, there should be 2500 responses in total. As a result, we received 3177 valid feedback (90.77\%), which means each participant responded 31.77 times on average.

The results of the analysis (Model 1 and Model 3) are showed in Table 1. As showed in Table 1, we found that at level 1 in model 1, the higher level of stress at the current time predicted increased negative emotions - depression, anger and anxiety - at the next time. At level 2, people who generally had higher level of stress were more possible to experience negative emotions in daily life, all of the depression, anger and anxiety.

The results of Model 2 at level 1 indicated that the higher level of stress at the current time can significantly predict higher level of rumination at the next time $\left(b_{10}=0.032, S E=0.016, p<0.05\right)$. At level 2 , the results showed that people who generally had higher level of stress tended to ruminate in daily life $\left(b_{01}=0.334, S E=0.040, p<0.001\right)$.

At last, the results of Model 3 showed in Table 1 indicated that rumination significantly mediated the relationship between stress and negative emotions. At level 1, people who had higher level of stress at the current time would experience higher rumination at the next time, and then experience more negative emotions (depression, anger, and anxiety). In other words, rumination mediated the relationship between stress and negative emotions dynamically. At level 2, the results showed that the individuals' overall rumination significantly mediated the relationship between overall stress and negative emotion (anger only) $\left(b_{01}=0.272, S E=0.123, p<0.05\right)$.

\section{Discussion}

In the current study, we investigate the associations between perceived haze, stress, and negative emotions in the daily life of college students in China. Consistent with previous studies, our findings showed that stress is positively associated with negative emotions $[35,36]$ as well as rumination $[37,38]$.

Additionally, we innovatively explored the predict relationship in a moment by moment view. Within-person model illustrated the dynamic influence that the higher level of stress at the current time predicted increased negative emotions - depression, anger and anxiety - at the next time. Besides, we found that people's momentary thinking of rumination positively predicted their experiences of next point negative emotions. More importantly, we found that rumination significantly mediated the relationship between stress and negative emotions in the two-time-point dynamic process. That is, the higher level of stress experienced, the higher level of rumination, which in turn predicted higher degree of negative emotions experienced. This 
Table 1. CWC(M) analysis for negative emotions, predicted by stress (Model 1) and stress and rumination (Model 3) at between-person level (level 1) and within-person level (level 2). CWC(M): centered within context with reintroduction of the subtracted means; SE: standard error.

\begin{tabular}{|c|c|c|c|c|c|c|c|c|c|c|}
\hline \multirow{3}{*}{$\begin{array}{l}\text { Negative } \\
\text { Emotions }\end{array}$} & \multirow{3}{*}{ Level } & \multirow{2}{*}{\multicolumn{3}{|c|}{$\begin{array}{c}\text { Model } 1 \text { (Stress) } \\
\text { Stress }\end{array}$}} & \multicolumn{6}{|c|}{ Model 3 (Stress and Rumination) } \\
\hline & & & & & \multicolumn{3}{|c|}{ Stress } & \multicolumn{3}{|c|}{ Rumination } \\
\hline & & $B$ & $S E$ & $p$ & $B$ & $S E$ & $P$ & $B$ & $S E$ & $p$ \\
\hline \multirow[t]{2}{*}{ Depression } & level 1 & 0.074 & 0.015 & 0.000 & 0.065 & 0.011 & 0.000 & 0.295 & 0.041 & 0.000 \\
\hline & level 2 & 0.237 & 0.047 & 0.000 & 0.168 & 0.051 & 0.001 & 0.206 & 0.125 & 0.130 \\
\hline \multirow[t]{2}{*}{ Anger } & level 1 & 0.046 & 0.012 & 0.000 & 0.039 & 0.011 & 0.000 & 0.205 & 0.036 & 0.000 \\
\hline & level 2 & 0.201 & 0.042 & 0.000 & 0.111 & 0.045 & 0.016 & 0.272 & 0.123 & 0.030 \\
\hline \multirow[t]{2}{*}{ Anxiety } & level 1 & 0.074 & 0.015 & 0.000 & 0.090 & 0.016 & 0.000 & 0.267 & 0.036 & 0.000 \\
\hline & level 2 & 0.237 & 0.047 & 0.000 & 0.337 & 0.061 & 0.000 & -0.119 & 0.137 & 0.390 \\
\hline
\end{tabular}

pattern of finding may reflect an adoption of the emotion-focused strategy (refers to the thoughts and actions people use to manage distress) to cope with the stress [39]. Most previous studies considered the stress coping at a between-person level, while the current study profoundly revealed the dynamic regulations about how rumination covaries with stress and negative emotions at a within-person, which can improve and contribute to Lazarus and Folkman's [8] theory from a more specific context with high ecological validity.

Moreover, the between-person level analyses revealed that the moderating effect of overall rumination in the relationship between overall stress and anger was significant. One explanation for the mediating effect is that people predisposed toward rumination allocate more attention to provocations, and thus, cause greater anger. Additionally, rumination maintains physiological activation (e.g., cardiovascular recovery) and emotional arousal [40] in response to stressors. These may be the reason why rumination can increase and maintain anger. There is another possibility, as the network models Miller, Pedersen, Earleywine, and Pollock [40] suggested, repetitive provocations maintain the anger-related activation networks over time, and then the angry maintained.

However, the mediating effect of overall rumination in the relationship between overall stress and depression and anxiety is not significant. The reason of the non-significant results may be that the predictive relationship between stress and anxiety and depression is already strong. Additionally, the repeated reflection on negative experiences may also lead to some positive understanding which may cause decreases in anxiety and depression. Finally, the participants are healthy college students, and they may have relatively low level of depression and anxiety.

The findings had significant implications for theories that attempted to explain the relationship between stress and negative emotion via rumination. First, this study was the first to used AA to investigate the relationship between stress at one time point and negative emotion at the next time point via rumination. Second, the current findings, which derived from more naturalistic and repeated measurements, provided complementary and persuasive evidence to the existing research. Finally, the current findings can also be effectively applied to mental health awareness programs in daily life contexts, to help high level stress people reduce negative emotions and maintain psychological health.

This study has some limitations that can be addressed in future research. First, the generalization of study should be cautious since our sample comprised only healthy college students in China. Second, this study has only examined the role of rumination as one potential mediator of the association between stress and negative emotions, the mechanisms underlying this association may be complicated. Future research should consider a number of other potential mediators of the above-mentioned association.
In conclusion, this study used ambulatory assessment to investigate one's level of rumination was one driver behind the relationship between perceived stress and experience of negative emotions in daily life. These results might help future research to explore whether these programs help people notice and stop rumination before it exacerbates negative moods.

\section{Acknowledgement}

This research was supported by the the 13th Five-Year Plan of Educational Science in Jiangsu Province (project no. C-c/2016/01/13), the Project of Philosophy and Social Science Research in Colleges and Universities in Jiangsu Province (project no. 2017SJB0218), and the Natural Science Foundation of Jiangsu Province of China (project no. BK20171036).

\section{References}

1. Folkman S (1997) Positive psychological states and coping with severe stress. Social science \& medicine 45: 1207-1221.

2. Folkman S1 (2008) The case for positive emotions in the stress process. Anxiety Stress Coping 21: 3-14. [Crossref]

3. Lazarus RS, Folkman S (1984) Coping and adaptation. The handbook of behavioral medicine pp. 282-325.

4. Egede LE, Zheng D, Simpson K (2002) Comorbid depression is associated with increased health care use and expenditures in individuals with diabetes. Diabetes care 25: 464-470.

5. Grigsby AB, Anderson RJ, Freedland KE, Clouse RE, Lustman PJ (2002) Prevalence of anxiety in adults with diabetes: a systematic review. Journal of psychosomatic research 53: 1053-1060.

6. Lave JR, Frank RG, Schulberg HC, Kamlet MS (1998) Cost-effectiveness of treatments for major depression in primary care practice. Archives of General Psychiatry 55: 645-651.

7. Lazaru RS (1966) Psychological stress and the coping process. APA Psyc NET, New York, McGraw-Hill.

8. Lazarus RS, Folkman S (1984) Coping and adaptation. The handbook of behavioral medicine pp. 282-325.

9. Kiecolt-Glaser JK, McGuire L, Robles TF, Glaser R (2002) Emotions, morbidity, and mortality: new perspectives from psychoneuroimmunology. Annual review of psychology 53: 83-107.

10. Sternberg EM, Chrousos GP, Wilder RL, Gold PW (1992) The stress response and the regulation of inflammatory disease. Annals of Internal Medicine 117: 854-866.

11. Fiedler N, Laumbach R, Kelly-McNeil K, Lioy P, Fan ZH, et al. (2005) Health effects of a mixture of indoor air volatile organics, their ozone oxidation products, and stress. Environmental health perspectives 113: 1542.

12. Aseltine RH, Gore S, Gordon J (2000) Life stress, anger and anxiety, and delinquency: An empirical test of general strain theory. Journal of Health and Social Behavior pp. 256-275.

13. Lee PS, Sohn JN, Lee YM, Park EY, Park JS (2005) A correlational study among perceived stress, anger expression, and depression in cancer patients. Journal of Korean Academy of Nursing 35: 195-205.

14. Shiffman S1, Stone AA, Hufford MR (2008) Ecological momentary assessment. Annu Rev Clin Psychol 4: 1-32. [Crossref] 
15. Trull TJ, Ebner-Priemer U (2014) The role of ambulatory assessment in psychological science. Curr Dir Psychol Sci 23: 466-470.

16. Fritz HL (1999) Rumination and adjustment to a first coronary event. Psychosomatic Medicine 61: 105.

17. Ciarocco NJ, Vohs KD, Baumeister RF (2010) Some good news about rumination: Task-focused thinking after failure facilitates performance improvement. Journal of social and clinical psychology 29: 1057-1073.

18. Watkins ER (2008) Constructive and unconstructive repetitive thought. Psychol Bull 134: 163-206. [Crossref]

19. Nolen-Hoeksema S (2004) The response styles theory. Depressive rumination: Nature, theory and treatment pp. 105-123.

20. Deyo M, Wilson KA, Ong J, Koopman C (2009) Mindfulness and rumination: does mindfulness training lead to reductions in the ruminative thinking associated with depression? Explore (NY) 5: 265-271.

21. Broderick PC (2005) Mindfulness and coping with dysphoric mood: Contrasts with rumination and distraction. Cognitive Therapy and Research 29: 501-510.

22. Just N, Alloy LB (1997) The response styles theory of depression: tests and an extension of the theory. Journal of abnormal psychology 106: 221.

23. Spasojevic J, Alloy LB (2001) Rumination as a common mechanism relating depressive risk factors to depression. Emotion 1: 25.

24. Treynor W, Gonzalez R, Nolen-Hoeksema S (2003) Rumination reconsidered: A psychometric analysis. Cognitive therapy and research 27: 247-259.

25. Bushman BJ (2002) Does venting anger feed or extinguish the flame? Catharsis, rumination, distraction, anger, and aggressive responding. Personality and social psychology bulletin 28: 724-731.

26. Rusting CL, Nolen-Hoeksema S (1998) Regulating responses to anger: effects of rumination and distraction on angry mood. Journal of personality and social psychology 74: 790 .

27. Nolen-Hoeksema S (2000) The role of rumination in depressive disorders and mixed anxiety/depressive symptoms. Journal of abnormal psychology 109: 504

28. Muris P, Roelofs J, Rassin E, Franken I, Mayer B (2005) Mediating effects of rumination and worry on the links between neuroticism, anxiety and depression. Personality and Individual Differences 39: 1105-1111.
29. De Raedt R, Koster EH (2010) Understanding vulnerability for depression from a cognitive neuroscience perspective: A reappraisal of attentional factors and a new conceptual framework. Cogn Affect Behav Neurosci 10: 50-70.

30. Koster EH, De Lissnyder E, Derakshan N, De Raedt R (2011) Understanding depressive rumination from a cognitive science perspective: The impaired disengagement hypothesis. Clinical psychology review 31: 138-145.

31. Whitmer AJ, Banich MT (2007) Inhibition versus switching deficits in different forms of rumination. Psychological science 18: 546-553.

32. Skitch SA, Abela JR (2008) Rumination in response to stress as a common vulnerability factor to depression and substance misuse in adolescence. Journal of Abnormal Child Psychology 36: 1029-1045.

33. Smith JM, Alloy LB (2009) A roadmap to rumination: A review of the definition, assessment, and conceptualization of this multifaceted construct. Clinical psychology review 29: 116-128.

34. Zhang Z, Zyphur MJ, Preacher KJ (2009) Testing multilevel mediation using hierarchical linear models: Problems and solutions. Organizational Research Methods 12: 695-719.

35. Kiecolt-Glaser JK, McGuire L, Robles TF, Glaser R (2002) Emotions, morbidity, and mortality: new perspectives from psychoneuroimmunology. Annual review of psychology 53: 83-107.

36. Smith JM, Alloy LB (2009) A roadmap to rumination: A review of the definition, assessment, and conceptualization of this multifaceted construct. Clinical psychology review 29: 116-128.

37. Skitch SA, Abela JR (2008) Rumination in response to stress as a common vulnerability factor to depression and substance misuse in adolescence. Journal of Abnormal Child Psychology 36: 1029-1045.

38. Folkman S, Moskowitz JT (2000) Positive affect and the other side of coping. Am Psychol 55: 647-654. [Crossref]

39. Denson TF (2013) The multiple systems model of angry rumination. Personality and Social Psychology Review 17: 103-123.

40. Miller N, Pedersen WC, Earleywine M, Pollock VE (2003) A theoretical model of triggered displaced aggression. Personality and Social Psychology Review 7: 75-97.

Copyright: @2018 Jiaxuan Du. This is an open-access article distributed under the terms of the Creative Commons Attribution License, which permits unrestricted use, distribution, and reproduction in any medium, provided the original author and source are credited. 